\title{
2D:4D Ratio in Adult Female and Their Comparison with Males: An Anthropometric Study from Northern India
}

\author{
Anamika Jaiswal, Ankit Kaushik*, A K Singh \\ ${ }^{1}$ Department of Anatomy, Government Medical College, Haldwani, Uttarakhand, India. \\ ${ }^{2}$ Department of Pathology, Government Medical College, Haldwani, Uttarakhand, India.
}

\begin{abstract}
Background: 2D:4D ratio is a sexually dimorphic marker that act as potential marker of prenatal androgen exposure. Genital development in fetus depends on adequate and timely exposure to sex steroids. Assessment of fetal androgen levels and exposure are essential to predict normal development and future reproductive disorders. The 2D:4D ratio can be used to assess androgen action.

Aim: This study attempts to find out 2D:4D ratio of left and right hand in adult females and males and to find out whether any difference exists among these variables in males and females.

Methods: A total of 83 adults were included in the study. There were 51 females and 32 males. The distance of second finger and fourth finger was calculated from mid-point of proximal most digital crease to distal most end of the finger. The ratio was then obtained by dividing second finger length by fourth finger length.

Result: The mean 2D:4D ratio of left hand and right hand in adult female were 0.98 and 0.99 respectively. The mean 2D: 4D ratio of left hand and right hand in adult male were 0.967 and 0.99 respectively. The males have lower 2D:4D ratio for left and right hand as compared to females. The mean 2D:4D ratio of left hand and right hand in left handed females were 0.99 and 1.01 respectively while mean 2D:4D ratio of left hand and right hand in right handed females were 0.987 and 0.99 . The mean 2D:4D ratio of left hand and right hand in left handed males were 0.96 and 0.98 respectively while mean 2D:4D ratio of left hand and right hand in right handed males were 0.98 and 1.01 .

Conclusion: Sexually dimorphic markers are variables that can be used as indirect marker of fetal androgen exposure. In this study the mean 2D:4D ratio in males for both left and right hand was lower than females in overall group and in case of both right and left handedness males and females. Male fetuses with higher testosterone levels will have a lower 2D:4D ratio. Digital ratio is an easy noninvasive parameter, abnormal 2D:4D ratio in females can predict excessive androgen action.
\end{abstract}

Keywords: 2D:4D Ratio, Sexual Dimorphism

\section{Introduction}

2D:4D ratio is a sexually dimorphic marker that act as potential marker of prenatal androgen exposure. It is the ratio of length of second finger to that of fourth finger. ${ }^{1}$ The dimorphism trait was found in various species including mice, zebra and humans. ${ }^{2}$ Among humans marked variations have been reported among different geographic region and various ethnic groups. ${ }^{3}$ Genital development in fetus depends on adequate and timely exposure to sex steroids. Various congenital abnormalities and reproductive disorders in adults are associated with abnormal fetal androgen exposure. ${ }^{4}$ Assessment of fetal androgen levels and exposure are essential to predict normal development and future reproductive disorders. The sexually dimorphic markers variables like 2D:4D ratio can be used to assess androgen action. ${ }^{5}$ These markers are different in males and females. Various studies done have given variable 2D:4D ratio among males and females from different region and ethninicity. ${ }^{3,6}$ This study attempts to find out 2D:4D ratio of left and right hand in adult females and males and to find out whether any difference exists among these variables in males and females.

\section{Materials and Methods}

This cross sectional study was done in department of Anatomy, Government Medical College, Haldwani, Nanital, Uttarakhand. A total of 83 adults were included in the study. There were 51 females and 32 males. The distance of second finger (index) and fourth finger (ring) was calculated from mid-point of proximal most digital crease to distal most end of the finger with the help of Vernier caliper. ${ }^{7}$ The ratio was then obtained by dividing second finger length by fourth finger length. The ratio was calculated for both right and left hand. The mean 2D:4D ratio in both males and females were calculated for both hands. The digital ratio of left hand of males and females were compared to find out, if there is any difference between male and female digital ratio of each hand. 


\section{Result}

The study included 83 adults with age range of 18 years to 20 years. There were 32 males and 51 females. The mean 2D:4D ratio of left hand and right hand in adult female were 0.98 and 0.99 respectively. The mean 2D: $4 \mathrm{D}$ ratio of left hand and right hand in adult male were 0.967 and 0.99 respectively. The standard deviation of left and right 2D:4D ratio in males were 0.027 and 0.032 while standard deviation of left and right hand in females were 0.03 for both the hands. The males have lower 2D:4D ratio for left and right hand as compared to females.(Table 1)(Graph 1)

The handedness was also observed in males and females. Out of 83 adults, 75 were right handed and 8 were left handed. Out of 8 left handed adults, 4 were males and rest were female. Out of 75 right handed adults, 47 were females and 28 were males.

The mean 2D:4D ratio of left hand and right hand in left handed females were 0.99 and 1.01 respectively while mean 2D:4D ratio of left hand and right hand in right handed females were 0.987 and 0.99.(Graph 2)

The mean 2D:4D ratio of left hand and right hand in left handed males were 0.96 and 0.98 respectively while mean 2D:4D ratio of left hand and right hand in right handed males were 0.98 and 1.01.(Graph 3)

\section{Discussion}

Early fetal reproductive development depends on adequate and timely androgen exposure. In initial weeks of development in sex determining region (SRY) on Y

Table 1: Shows mean and standard deviation of 2D:4D ratio of left and right hand in males and females.

\begin{tabular}{|l|c|c|c|c|} 
& Left(Male) & Right(Male) & Left(Female) & Right(Female) \\
\hline Mean & 0.967 & 0.97 & 0.987 & 0.99 \\
\hline Stand dev & 0.027 & 0.032 & 0.03 & 0.03 \\
\hline
\end{tabular}

Table 2: Shows mean 2D:4D ratio of left and right hand in left and right handed males and females.

\begin{tabular}{|c|c|c|c|c|}
\hline Sex & Left hand right handed & Left hand left handed & Right hand left handed & Right hand Right handed \\
\hline M & 0.967 & 0.96 & 0.98 & 0.969 \\
\hline F & 0.987 & 0.982 & 1.01 & 0.99 \\
\hline
\end{tabular}

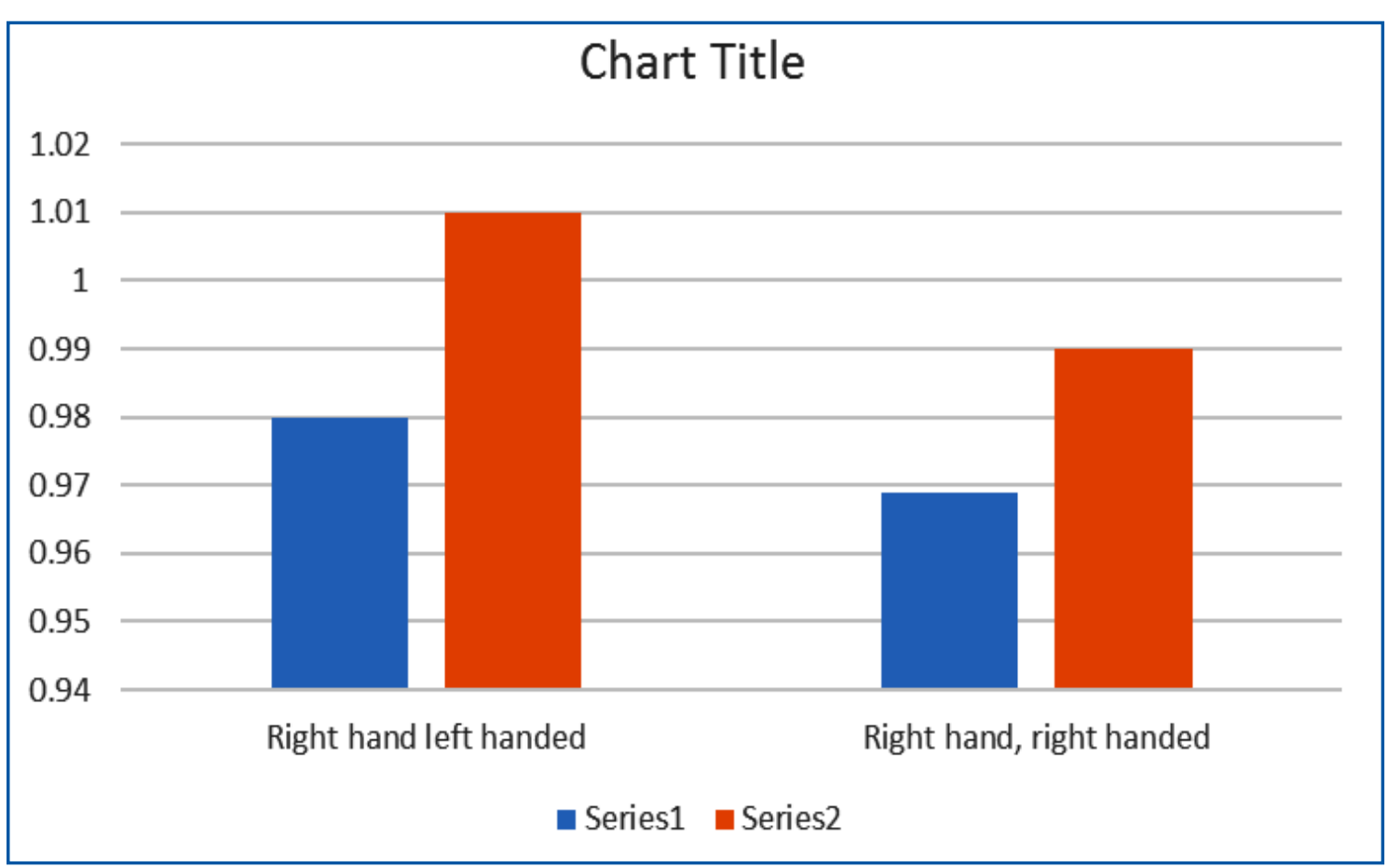

Graph 1: Shows mean 2D:4D ratio of left and right hand in males(Blue) and females(Orange). The males have lesser 2D:4D ratio then females. 


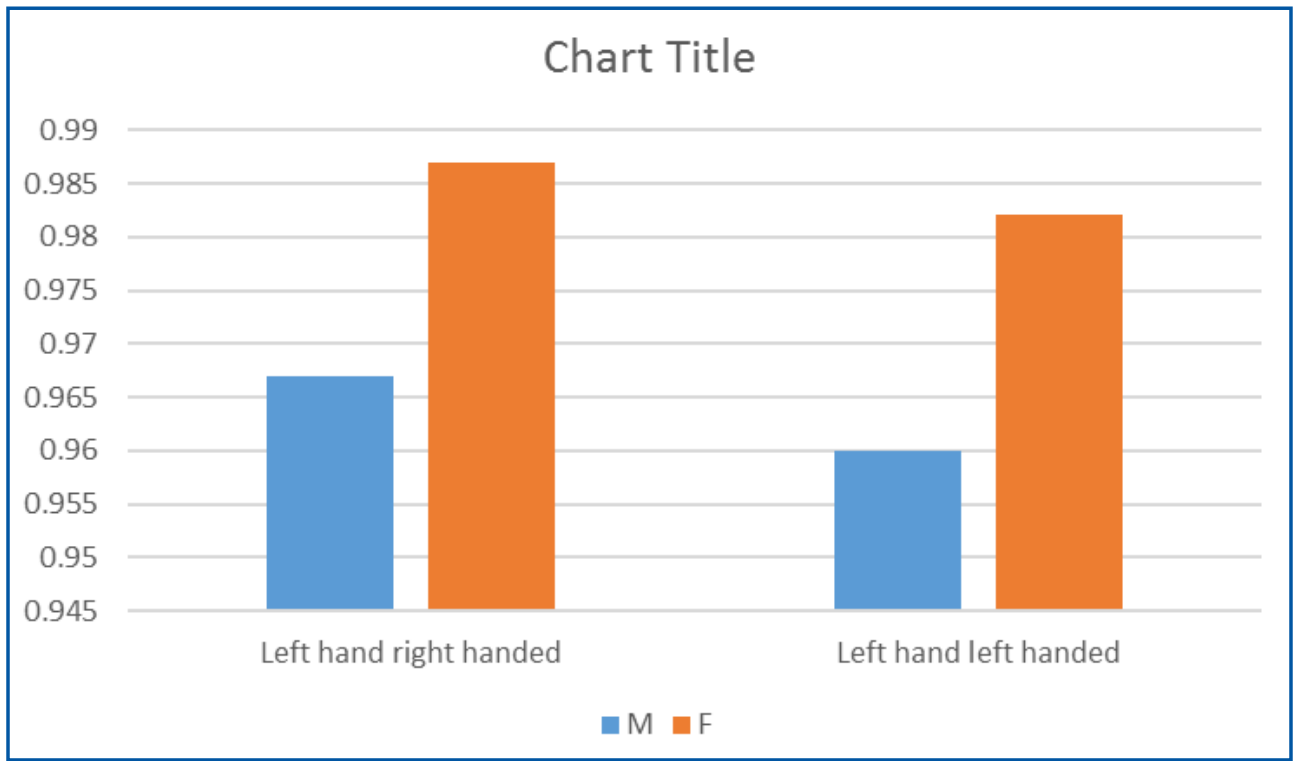

Graph 2: Shows mean 2D:4D ratio in males(blue) and females(orange). The males have lesser 2D:4D ratio in left hand for both right and left handed in males and females.

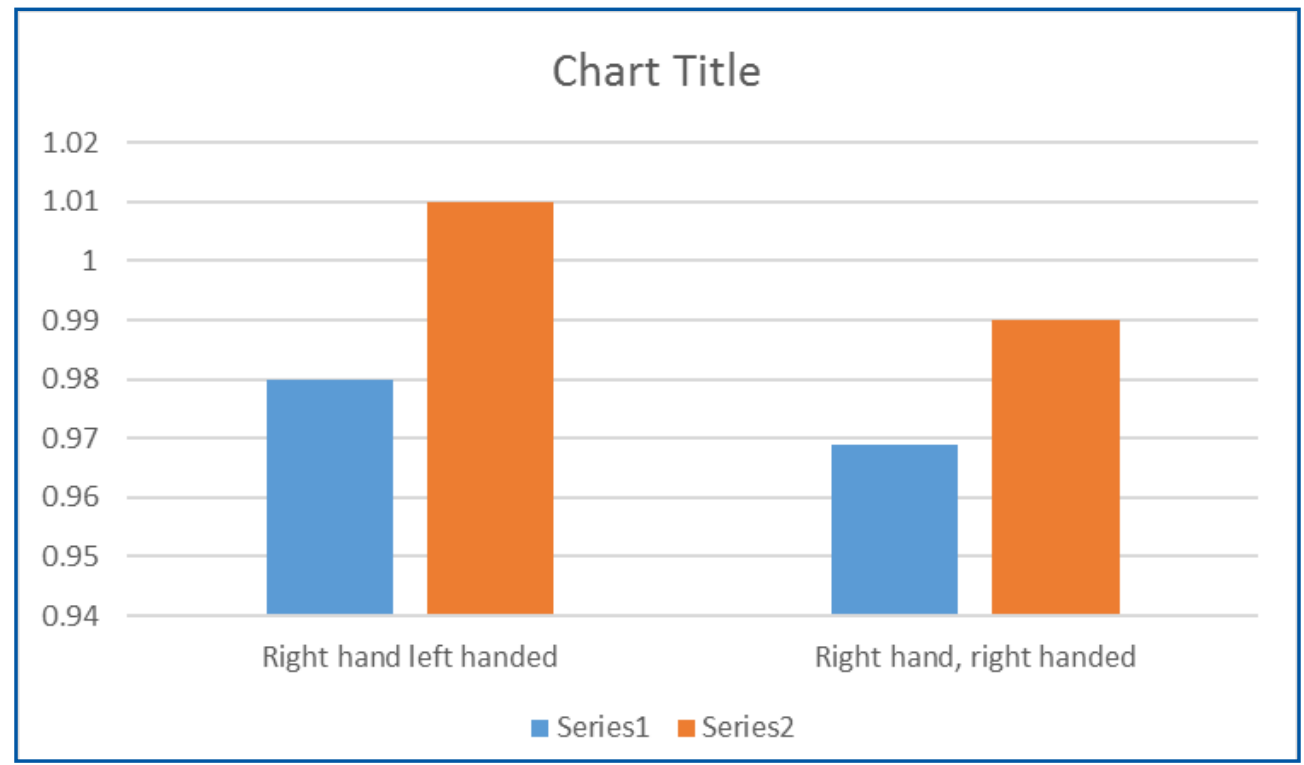

Graph 3: Shows mean 2D:4D ratio in males(blue) and females(orange). The males have lesser 2D:4D ratio in right hand for both right handed and left handed males and females.

chromosomes leads to differentiation of leydig cells, these cells start production of testosterone. ${ }^{8}$ In presence of testosterones the mullerian duct degenerates and male internal genitalia develops from wollfian duct. The male external genitalia develop from genital tubercle in presence of 5DHT, a derivative of testosterone. In absence of testosterone, female internal genitalia develop from mullerian duct and clitoris develops from genital tubercle. ${ }^{9,10}$ Therefore, timely and adequate exposure of androgens are needed for proper genital development. Inadequate and excessive exposure in fetus can leads to various congenital abnormalities and reproductive disorders in adults. ${ }^{11}$ Therefore, assessment of fetal androgens levels and action are necessary to rule out future reproductive disorders. Direct assessment of androgens levels is not possible in fetus and indirect measurement of fetal androgen action are necessary for the same. Sexually dimorphic markers are variables that can be used as indirect marker of fetal androgen exposure. 2D:4D ratio is ratio of length of second digit to the length of fourth digit. 
In this study the mean 2D:4D ratio in males for both left and right hand was lower than females in overall group and in case of both right and left handedness males and females. This lesser 2D:4D ratio in males and greater 2D:4D ratio in females were expressed as masculine and femine in males and females respectively.

The mean 2D:4D ratio in males for left and right hand was $0.967 \pm 0.027$ and $0.97 \pm 0.03$ while mean $2 \mathrm{D}: 4 \mathrm{D}$ ratio in females for left and right hand was $0.98 \pm 0.03$ and $0.99 \pm 0.03$. Loehlin $\mathrm{JC}$ etal ${ }^{12}$ in there study found that $2 \mathrm{D}$ : 4D ratios among males and females were lesserin males compared to females with values of $0.947 \pm 0.029$ and $0.965 \pm 0.026$, respectively.

Rebecca $\mathrm{B}^{13}$ in their study found that testosterone levels in fetus are negatively correlated with 2D:4D ratio while estrogen has positive correlation with estrogen. Therefore, male fetuses with higher testosterone levels will have a lower 2D:4D ratio, on the other hand females have lower levels of testosterone and have higher 2D:4D ratio. Jacob $\mathrm{M}$ eta $1^{14}$ in their study on South Indian population also found sexual dimorphism.

This difference in digit ratio were also correlated with various disorders that are associated with under and overexposure of androgen levels. The females with congenital adrenal hyperplasia(CAH) have a higher level of androgen exposure and therefore have a lower 2D:4D ratio. ${ }^{15}$ In a study by Coyne SM etal ${ }^{16}$ found that smoking in pregnancy is associated with higher testosterone levels and therefore the female fetuses have lower digital ratio. The ratio once established at birth remains same throughout the life and little influence by androgen levels in post-natal life. Various phenotypic traits were attributed to 2D:4D ratio including sperm count, personalities, handedness, spatial skills, academic performance, depression, autism etc. ${ }^{17}$

2D:4D ratio shows marked ethnic variations. Manning et $\mathrm{al}^{3}$ in his study among different ethnic study showed sexual dimorphism but the digital ratio showed marked variations among children of different ethnic communities. Xi H etal $^{6}$ in their study on children found higher ratio among caucasians then blacks and Hans ethnics of China. The relationship of 2D:4D ratio with metabolic syndrome and cardiovascular risk factors were also studied. ${ }^{18}$

Digital ratio is an important noninvasive sexually dimorphic marker. Abnormal values can be associated with congenital reproductive abnormality. This marker has great potential role to determine androgen exposure in fetal life and reproductive disorders in adult females. The use is limited by fact that it shows large geographical, ethical and regional variations. This study is a step forward in this regard and shows digital ratio from Northern India.

\section{Conclusion}

2D:4D ratio is a sexually dimorphic marker that can be used as indirect marker for androgen levels and action in fetal life, this study showed that males have lesser 2D:4D ratio in both hands as compared to females. In both right and left handed males and females, males were found to have lesser digital ratio. Digital ratio is an easy noninvasive parameter, abnormal 2D:4D ratio in females can predict excessive androgen action.

\section{Reference}

1. Mayhew TM, Gillam L, McDonald R, Ebling FJ. Human 2D (index) and 4D (ring) digit lengths: their variation and relationships during the menstrual cycle. Journal of Anatomy. 2007;211(5):630-38.

2. Bailey Allison A, Hurd PL. Depression in men is associated with more feminine finger length ratios. Personality and Individual Differences. 2005;39(4):829-36.

3. Manning JT, Stewart AG, Bundred P, Trivers R. Sex and ethnic differences in 2nd to 4th digit ratio of children. Early Human Development. 2004;80(2):161-68.

4. Thankamony A, Pasterski V, Ong KK, Acerini CL, Hughes IA. Anogenital distance as a marker of androgen exposure in humans. Andrology. 2016;4:616-25.

5. Dean A, Sharpe RM. Clinical review: Anogenital distance or digit length ratio as measures of fetal androgen exposure: relationship to male reproductive development and its disorders. J Clin Endocrinol Metab. 2013;98(6):2230-8.

6. Xi H, Li M, Fan Y, Zhao L. A comparison of measurement methods and sexual dimorphism for digit ratio (2D: 4D) in Han ethnicity. Archives of Sexual Behavior.2014;43(2):329-33.

7. Jeevanandam S, Prathibha K Muthu PK. 2D:4D Ratio and its Implications in Medicine.J Clin Diagn Res. 2016 Dec; 10(12): CM01-CM03.

8. Sinclair AH, Berta P, Palmer MS, Hawkins JR, Griffiths BL, Smith MJ, et al. A gene from the human sex-determining region encodes a protein with homology to a conserved DNA-binding motif. Nature. 1990;346(6281):240-4.

9. Blaschko SD, Cunha GR, Baskin LS. Molecular mechanisms of external genitalia development. Differentiation. 2012 Oct;84(3):261-8.

10. Wilson JD, Griffin JE, Leshin M, George FW. Role of gonadal hormones in development of the sexual phenotypes. Hum Genet. 1981;58(1):78-84.

11. Goldman AS, Bongiovanni AM. Induced genital anomalies. Ann N Y Acad Sci. 1967;142:755-67.

12. Loehlin JC, Medland SE, Martin NG. Is CAG sequence length in the androgen receptor gene correlated with finger-length ratio? Personality and Individual Differences. 2012;52(2):224-27. 
13. Rebecca B, Benson PJ. Digit ratio (2D: 4D) and the spatial representation of magnitude. Hormones and Behavior. 2006;50(2):194-9.

14. Jacob M, Avadhani R, Nair B, Nallathamby R, Soman MA. Cross sectional study of second and fourth digit ratio with physical attributes in South Indian population. Int $\mathrm{J}$ Anat Res. 2015;3(2):1133-7.

15. Ciumas C, Lindén Hirschberg A, Savic I. High fetal testosterone and sexually dimorphic cerebral networks in females. Cereb Cortex. 2009;19(5):1164-72.

16. Coyne SM, Manning JT, Ringer L, Bailey L. Directional asymmetry (right-left differences) in digit ratio (2D: 4D) predict indirect aggression in women. Personality and Individual Differences. 2007;43(4):865-72.

17. Trivers R, Manning J, Jacobson A. A longitudinal study of digit ratio (2D: 4D) and other fin-ger ratios in Jamaican children. Hormones and Behavior. 2006;49(2):150-56.

18. Oyeyemi BF, Iyiola OA, Oyeyemi AW, Oricha KA, Anifowoshe AT, Alamukii NA. Sexual dimorphism in ratio of second and fourth digits and its relationship with metabolic syndrome indices and cardiovascular risk factors. Journal of Research in Medical Sciences: The Official Journal of Isfahan University of Medical Sciences. 2014;19(3):234-9.

*Corresponding author:

Dr Ankit Kaushik, Assistant Professor, Department of Pathology, Government Medical College, Haldwani, Uttarakhand, India,

Phone: +91 9953816240

Email: kaushikankit30@yahoo.co.in 